\title{
Dental Implant Abutments and Their Selection - A Review
}

\author{
Veena Benakatti1 ${ }^{1}$, Jayashree Arun Sajjanar², Aditya Ramnarayan Acharya ${ }^{3}$ \\ ${ }^{1,3}$ Department of Prosthodontics and Crown and Bridge, KAHER's KLE VK Institute of Dental \\ Sciences, Belagavi, Karnataka, India, ${ }^{2}$ Department of Prosthodontics, Swargiya Dadasaheb \\ Kalmegh Smruti Dental College and Hospital, Nagpur, Maharashtra, India.
}

\section{ABSTRACT}

Dental implants have become the most promising and accepted prosthetic alternative to missing teeth. Innovative and technological advances in implant dentistry have improved the performance and long term prognosis of dental implants. The growing demand for dental implants has led many manufacturers to enter the industry. With this growth in implant dentistry various implant brands and their components are made available in the markets that vary in terms of design and other features. Success of implant restorations is related to various components involved with the prosthesis. Implant abutment is the link between implant and restoration; right selection of this abutment is vital for the success of implant prosthesis.

There are varieties of implant abutments available in the market. Clinician should have adequate knowledge about these abutments and various factors that affect abutment selection, to choose the right one. Each implant case is different and clinician should be able to identify requirements of every individual case and choose the best suitable abutment available in the market. These abutments vary in implant abutment connection, material, type of retention, and fabrication methods. Though the ideal concept is prosthetically driven implant placement, many times anatomical features, esthetic requirements, soft tissue profile, axial load distribution, malpositioned implants, and many other patient factors do not allow ideal implant placement.

Such cases can be managed by careful selection of abutments by assessing the clinical requirements, thus offering better treatment outcome. This paper is an attempt to contemplate various factors affecting abutment selection, and throw light on various implant abutments available in the market, thus guiding the clinician in abutment selection. Hence this article summarizes complete information about the implant abutments, including introduction, types, selection, and different abutments available in market.

\section{KEY WORDS}

Abutment Material, Implant Abutments, Implant Abutment Connection, Prosthesis Retention
Corresponding Author: Dr. Veena Benakatti, Department of Prosthodontics and Crown and Bridge, Kaher's KLE VK Institute of Dental Sciences, Neharu Nagar - 590010, Belagavi,

Karnataka, India.

E-mail: veenak20021@rediffmail.com

DOI: $10.14260 / \mathrm{jemds} / 2021 / 622$

How to Cite This Article:

Benakatti V, Sajjanar JA, Acharya AR. Dental implant abutments and their selection - a review. J Evolution Med Dent Sci 2021;10(35):3053-3059, DOI: $10.14260 /$ jemds/2021/622

Submission 20-04-2021,

Peer Review 24-06-2021,

Acceptance 02-07-2021,

Published 30-08-2021.

Copyright (c) 2021 Veena Benakatti et al. This is an open access article distributed under Creative Commons Attribution License [Attribution 4.0 International (CC BY 4.0)] 


\section{BACKGROUND}

Implantology is a prosthodontically driven subject with surgical component. Prosthesis will determine where the implant should ideally be placed. Successful implant restoration depends on various factors, case selection, implant placement, osseointegration, abutment selection and maintenance. Abutment selection is an extremely important step to achieve functional and esthetic harmony in implant prosthesis. The abutment is the portion of the implant that supports or retains prosthesis or implant super structure. A superstructure is a metal framework that attaches to the implant abutment and provides either retention for a removable prosthesis or acts as framework for fixed prosthesis. ${ }^{1}$

The implant abutment has three parts; Prosthesis connection segment that connects to prosthesis, Implant connection segment that connects to implant and Transgingival segment that is surrounded by gingival tissue above prosthetic platform of implant. The restoration can be connected to implant in three ways referred to as implant tier system namely, two tier system and three tier system.

Two tier system comprises of two individual components, implant as one component, abutment and crown as one component or conversely implant and abutment in one component and crown in one component. Three tier systems constitute implant, abutment and crown as individual components. ${ }^{2}$ Dental implantology has evolved over five decades in terms of material science, design and surface topography, restorative techniques and components to meet demands of changing clinical scenarios. There are over 220 implant manufacturers in the world and they provide large variety of implants and its components to comply with divergent clinical situations. This puts the clinician in dilemmatic situation in regard to abutment selection to accomplish satisfactory outcome especially if implant is placed in compromised position. ${ }^{3}$

This review paper unveils that there are many articles concerning implant dentistry. However, there is a need for an in depth review on implant abutments and their selection. Hence this article summarizes complete information about implant abutments including introduction, types, selection and different abutments available in the market.

\section{Selection of Implant Abutments}

Selection of implant abutments depend on various factors and varies on case to case basis. Clinician should have adequate knowledge about these factors affecting abutment selection and various implant abutments available in market to choose from. We have discussed about this aspect in the following section to help clinician in abutment selection.

\section{IMPLANT ABUTMENT CONNECTION}

The implant abutment connection region has a platform on which abutment is seated and this platform bears an antirotational feature to resist rotation between abutment and implant body. If this antirotational component is on implant platform on which abutment is seated, it is external hex, if this feature extends within the implant body it is internal hex. These antirotational features can take up various configurations namely, hexagonal, octagonal, tripod, spline and morse taper connections. ${ }^{1,3}$

Another class of implants without antirotational features has a flat surface generally used with one piece abutment. These implants are recommended in case of multiple units splinted by overlying crowns, consequently preventing abutment rotation. Implants with antirotational features are recommended in single tooth restorations to preclude rotation of abutments. Implant abutment connection may be slip fit wherein little space exists between mating parts, or friction fit where no space exists between parts. This interface may have a butt or beveled joint. ${ }^{3}$

When choosing implant abutment, the interface should essentially be secure, strong, passive and withstand screw loosening under function. ${ }^{4}$ Insecure joint not only leads to screw loosening but also results in plaque accumulation and altered soft tissue response. Hence, lesser ideal interface selection may result in substantial biologic and mechanical complications.

\section{External Hex}

External hex implants were first introduced by branemark and have served well over years. Merits of external hex are its applicability for two stage method, anti-rotational feature, retrievability and interchangeability among manufacturers. External hex has certain drawbacks viz, micro movements between mating parts, less resistance to lateral and rotational movements and micro gaps resulting in bone resorption. ${ }^{5}$

To overcome these drawbacks, several modifications of external hex, tapered external hexagon, external octagon and spline connection were made available.

\section{Internal Hex}

Advocates of this design claim that, this configuration decreases the height from implant platform to the abutment top, distribute the forces deep within implant providing better shield for the abutment screw and captures long internal walls creating a stable body to resist micro movement thus preventing micro leakage. This distinctive feature furnishes a stable joint, incorporates a tactile and audible click when the components are seated. ${ }^{5}$

Morse taper was introduced with an attempt to further enhance implant abutment connection, wherein tapered projection from the abutment was inserted into corresponding recess within implant. This internal connection resulted in friction fit and cold welding at interface enhancing resistance to bending forces, micromotion, micro leakage and abutment loosening. 3,5

Majority of the studies inferred that the internal hex with morse taper seems to be superior connection than external hex connections that completely depends on screw to hold abutment in position.3,5 Internal hex assumes a proper abutment seating, excellent esthetics, anti-rotational engagement, resistance to lateral forces and higher stability under loading conditions. 


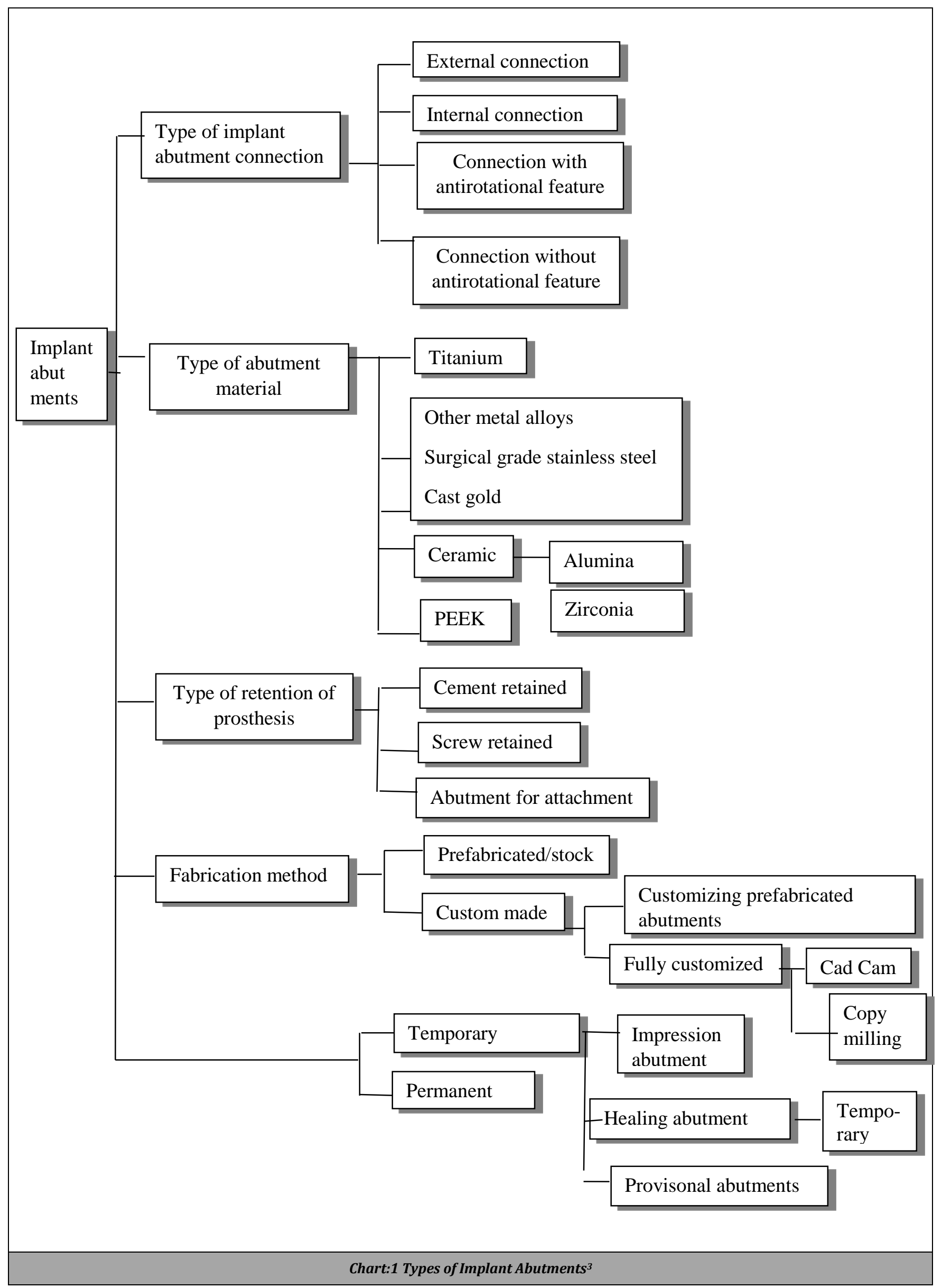




\section{BASED ON PROSTHESIS RETENTION}

Implant prosthesis can be retained by screw, cement or an attachment. There are various factors to be considered before selecting the mode of retention for the prosthesis. A comprehensive knowledge about their mechanism and function will help the operator to choose the right mode of retention. ${ }^{3}$

\section{Cost and Fabrication}

Cost is a factor when we decide to use an abutment, usually crown and prosthesis that connect to implant directly without an abutment are less expensive as they require lesser components and need less time for technician. Fabricating a cement retained restorations is more simpler as it employs conventional clinical and laboratory procedures hence costs less than screw retained restorations. Screw retianed restorations are comparatively expensive as it involves additional components and complex laboratory procedures for their fabrication. 6

\section{Aesthetics}

Screw and cement retained restorations both deliver enhanced esthetics if an ideal implant position is achieved. If unable to achieve ideal implant position, custom and angulated abutments can be employed so that access hole for screw can be repositioned away from esthetic zone. With cement retianed restorations esthetics is more predictable as it does not involve screw access holes. ${ }^{6}$

\section{Accessibilty}

Screw retained restorations are difficult to access in comparison to cement retained restorations, especially in posterior region of oral cavity and in cases of limited jaw opening. There is possibilty of swallowing or aspirating the screws and screw drivers with screw retained restorations. ${ }^{3,6}$

\section{Occlusion}

Achieving a more precise occlusion is possible with cement retained restorations as it lacks screw access holes. Screw access holes interfere with lateral and protrusive excursions making it difficult to establish occlusion. Screw access holes occupy more than $50 \%$ of occlusal table and need to be covered with occlusal restorative material to conceal screw channel. Under occlusal loading the restorative material tends to wear off abolishing the occlusal contacts, such is not the case with cement retained restorations. . $^{3,6}$

\section{Interocclusal Space}

The interocclusal space available for prosthesis affects the choice of abutment. Screw retained restorations that are connected to implants without use of intermediate abutment need less interocclusal space. When using an abutment, a minimum of $8 \mathrm{~mm}$ interocclusal space is required to achieve a good retention and esthetics. With cement retained restorations atleast $4 \mathrm{~mm}$ height for the abutment is required to achieve desirable retention. Hence cases with inadequate interocclusal space can be better managed with screw retained restorations. ${ }^{1,3}$

\section{Retention}

Principles of retention for cement retained implant restorations follow the principles of natural tooth preparation, taper, abutment height, roughness, surface area and type of cement. Abutments for cement retained restorations require a minimum of $4 \mathrm{~mm}$ height to retain the prosthesis, this becomes difficult in cases with compromised interocclusal space. Screw retianed retsorations are advantageous in such cases as they can be attached to implants directly without intermediate abutment. Screw loosening itself is a hindering factor in retention with screw retained restorations, hence a precise protocol has to be followed for tightening screws. After initial torquing, retorquing of screw after 5 minutes, and again after few weeks is recommended to overcome settling effect.6,7

\section{Retrievability}

Screw retained restorations can be easily retrieved without damaging the prosthesis and the implant thus enabling the future follow up and evaluation hassle free. Retrieving the cement retained restorations is possible but unpredictable and might cause damage to implant and restoration. Several modifications have been suggested to overcome the retrievability issue with cement retained restorations. Retrieving the abutment and restoration as one section by accessing abutment screw through access hole on occlusal surface is effective means to retrieve restoration without damaging implant body. Access hole is prepared on lingual side of restoration and cylindrical guide hole on lingual surface of the abutment, then driver is inserted into guide hole through an access hole and turned to initiate shear force, this loosens the cement and restoration can be easily retrieved.6,7

Cementation for the cement retained restorations can be achieved with either provisonal or a definitive cementation. Provisonal cementation offers a better retrivability but, exhibits high risk of micro leakage and retention loss in comparison to definitive cementation. Hence to achieve optimum retention with cement retained restorations, height and diameter of the abutment, conicity, surface roughness, indexing, alighnment of the abutments and straight or angled configurations play a crucial role. A cement gap plays an important role in seating of the restoration. When placing the restoration as cement sets, an error in proper seating of the restoration leads to loss of passivity of the restoration. If immediate retrieval is required due to improper seating, restoration retrieval could cause damage. These possibilties of errors and complications should be considered before choosing the implant abutments. ${ }^{8}$ Maintenance aspect of the implant prosthesis is favoured by easy retrivability of screw retained restorations. When considering aged population demanding for fixed prosthesis sometimes with long span or a full arch prosthesis, situation demands a meticulous hygiene maintenance. Even with prosthesis designed for easy cleansibilty, sometimes it remains difficult for patients to maintain hygiene. Then removal of the prosthesis for maintenance is a possible alternative with screw retention, thus preserving the health of peri implant mucosa. Therefore 
the approach of screw retention proves beneficial for elderly and special need patients. ${ }^{8}$

\section{Passive Prosthesis Fit}

A passive fit is desired for implant success, cement retained restorations achieve better passivity in comparison to screw retained restorations. Passivity is achieved by the cement that acts like a shock absorber and lower stress at implant abutment complex and bone. Without precise fit, screw retained restorations may generate significant stress within bone, implant and the prosthesis. However various methods have been proposed to improve passivity of screw retained as in laser welding of prosthesis framework and CAD CAM designing of restorations ${ }^{6}$ However, complete passive fit remains a difficult task to achieve.

\section{Complications}

Excess cement is an issue with cement retained restorations especially in anterior region when implant is placed $3-4 \mathrm{~mm}$ apically to achieve emergence profile. This excess cement can cause peri implantitis and related complications. In such scenarios restoration of choice is screw retained or customized restorations for cement retention with margin that follows natural gingival contour. Any attempt to remove excess cement with instrument or scaler may abrade implant surface resulting in plaque accumulation. However, excess cement is not a problem with screw retained restorations yet, screw loosening can cause granulation tissue formation, plaque accumulation and screw and porcelain fracture.6,7 To avoid problems with residual cement, screw retention is preferred in anterior sites, as the cement removal becomes difficult when implants are placed too deep within the tissue. With malpositioned implants, a combination of screw and cement retained restoration to customize cement margins offer a better outcome. ${ }^{8}$

\section{Provisonalization}

Screw retained restorations make an ideal choice for provisional restorations owing to their easy retrievability, better tissue response. Excess cement is again an issue with cement retained restorations. . $^{3,6}$

Screw retention is recommended as a primary mode of retention, as they can be easily retrieved and technical complications can be resolved better than the biologic complications found with cement retained restorations. ${ }^{7}$ Biologic complications if mismanaged may result in loss of implant. With prosthetic screw being the weakest component in implant restoration, any screw loosening will allow in evaluating the implant restoration before any significant complications arise, such as implant fracture at screw level mainly when using internal connections. ${ }^{6}$

Whenever cement retained restorations are chosen, abutment design plays a primary role, and poorly designed abutments lead to improperly contoured and unacceptable restorations leading to esthetic and mechanical complications. ${ }^{4}$ It is recommended that long span restorations be preferably screw-retained restorations for easier maintenance as they have high risk of complications. ${ }^{8}$

\section{ONE PIECE AND TWO PIECE ABUTMENT}

One piece abutment does not have additional retaining screw but connects to implant with friction or screw threads. Two piece abutment has two components, one engages antirotational feature and the other fixes abutment to implant. One piece implant is generally used with immediate and provisonal restorations. One piece abutments are structurally strong, require less components offering a precise control over final fitting of restoration and reduced cost. One piece abutment positioning procedure is complicated and may interfere with stabilty and osseointgration of implants. ${ }^{1}$

\section{TYPE OF MATERIAL}

The implant abutment emanates from implant platform and remians deep within soft tissue complex. Hence the abutment material should obviously be biocompatible with tissues. ${ }^{4}$ Currently used implant materials are titanium, zirconia, alumina, Peek, gold alloys and other metal alloys. Apart from biocompatibility these materials should also possess ideal mechanical properties to withstand occlusal load and survive in oral environment.

Titanium is the ideal implant material in regards to biocompatibility and mechanical properties, however with thin gingival biotype, titanium displays a greyish hue under tissues compromising esthetics especially in anterior region. This leads to introduction of esthetic ceramic materials that includes alumina and zirconia. Alumina exhibited good biocompatibility and esthetic outcome neverthless fracture at implant abutment junction was inevitable owing to its brittle nature. Zirconia showed high mechanical strength, excellent esthetics, biocompatibility and high loading capacity thus preferred over alumina. Zirconia also showed better soft tissue response than titanium. Abutment material is considered as main factor influencing the stability of peri implant mucosa and crestal bone. ${ }^{9}$

Zirconia is mechanically weaker than titanium, in high oclusal loading conditions as in posterior region, titanium is more ideal and in esthetic demanding areas like anterior region and low occlusal loading, zirconia is recommended. Adequate material thickness is a key factor for zirconia to achieve optimal performance. zirconia has to be avoided in high occlusal loading conditions with limited interocclusal distance. ${ }^{7}$

Several other alternatives like gold alloys, stainless steel, nickel and cobalt chrome alloys have been instituted for fabrication of abutments. These materials may lead to galvanic action because of dissimilar metals that can induce electrochemical corossion, oxidation and initiate pain. Owing to the gold price and advances in CAD CAM manufacturing, the trend shifted to non precious alternatives. Casting procedures adversely affect fitting surface and these abutments exhibit altered tissue response. ${ }^{3}$

Peek is a newer material introduced into dentsitry, it is chemically inert, colour compatible and has properties comparable to titanium. Peek is successfully used as healing abutment and also for provisonal restorations. ${ }^{3}$ Further research is required for Peek to be used as peramanent abutment and restoration. 
In cases with high esthetic situation, high smile line, anatomically demanding situations like triangular teeth and high scalloping of tissues, and mechanically low challenges, customized all ceramic abutments are recommended. In mechanically challenging situations with low esthetic demand, metallic abutments are recommended. In esthetically demanding situations which are mechanically challenging, hybrid abutments with titanium base are a good option. ${ }^{7}$

Soft tissue discoloration is evident with metallic abutments, peek and ceramic components exhibit less discolouration in comparison to metal. In cases with thin gingival biotype, tooth coloured materials like peek and

\section{Prefabricated Abutments}

The first branemark abutment consisted of a hollow cylinder with internal hex fitted to implant and was secured with an abutment screw. This standard abutments available in different range of collar heights to harmonize with subgingival position of implant. The first abutment designs were the threaded tapered posts that were hand tightened or ratcheted within the implant body. Other approach was to cement abutment post into implant body. These abutments had limited applications due to esthetic concerns. ${ }^{10}$

With invent of plastic UCLA abutments there was a significat breakthrough in implant restoration with an improvement in esthetics. This abutment is a castable abutment that comes with a machined gold cylinder and a plastic sleeve which can be modified to custom abutment.This abutment sleeve is then waxed up in the lab to a core. This allows to compensate for wide range of tooth size and angulations with a machined fitting surface to make it certain that abutment fits accurately to implant. Restoration can then be completed with conventional technique. UCLA abutment can be used for single and multi-unit screw or cement-retained restorations. It can correct up to 30 degree angulations when cast as a custom abutment. 10

Soon the angulated abutments became available that could correct offsets ranging from 15 - 35 degree. Use of angled abutments are limited by the angulations available, large and bulbous collars, and the required vertical height of the restoration. A range of collar heights are available for these abutments allowing selection based on mucosal thickness. ${ }^{10}$

In most cases these prefabricated abutments would still require some modifications like height and wall alighnment corrections which can be done chairside or in lab. To overcome esthetic issues, manufacturers incorporated gold coloured abutment surfaces to mask greyish discoloration under thin gingival biotype. Prefabricated abutments offer several advantages mainly the cost and ease of usage, however challenge lies with diameter of loading platform and emergence profile. Transition from implant to cervical area of crown is better controlled by custom abutment. Hence prefabricted abutments have limited application in anterior esthetic zone but offer best application in posterior area of oral cavity. ${ }^{10}$ Prefabricated / stock abutments cannot remain deep in the margin without being too deep interproximally, hence customised abutments have a better application in highly scalloped gingival margins. ${ }^{7}$

Custom abutments provide freedom to individualize position, angulations and emergence profile. Crown margins can be customized to follow gingival contour providing better ceramics are preferred to avoid discoloration and greyish hue display, thus achieving better esthetics.

\section{METHOD OF FABRICATION}

Implant abutments can be prefabricated or customised. Prefabricated or stock abutments are fabricated by the manufacturer and prosthesis is directly fixed to the abutment. Custom abutments are customised according to the individual needs either in the laboratory or chairside.

soft tissue support. Hence, custom abutments are the choice in optimal esthetics. Custom abutments on overall provide good combination of esthetics and strength. Custom abutments are advisable in anatomically compromised situations, combined with high esthetic demands and high smile line conditions. Custom abutments can either be fabricated in the laboratory, or by a copy milling procedure and CAD-CAM technology. CADCAM abutments are the ones designed by a computer and machined to exact requirements. Based on implant impression CAD CAM system permits to design individual abutments or by scanning of abutment wax up. This is a one step process that eliminates the necessity to buy stock abutment and modify in lab. CAD-CAM abutments extend the benefits of precise fit, ideal emergence profile, improved esthetics and proper restoration contours. ${ }^{11}$

In copy milling, first the abutment is manually prepared with composite or wax and then it is placed in pantographic machine. Copy milling machine has two arms, the copying arm traces the abutment pattern and the cutting arm which has a cutter, mills selected zirconia / metal block. With correct use of this technique, abutment fabrication be well simplified. Copy milled abutments can be fabricated in alumina, zirconia or titanium using scanner and CAM. ${ }^{12}$

\section{Multiunit Abutments}

The angulations and depth of implants will often vary in fullarch cases, which complicates restorative phase of treatment and impacts the design of the prosthesis. In addition to correcting implant angulations, multi-unit abutments can ease the delivery of the restoration, raise the implant connection close to the gingival surface, and create a level prosthetic platform. Considering all the time and resources, multi-unit abutments represent a modest investment towards an efficient, accurate and predictable restoration. Multi unit abutments are designed for fixed screw-retained restoration of complete and partially edentulous arches including All-on4 concept. It comes in straight and angled $\left(17^{\circ}\right.$ and $\left.30^{\circ}\right)$ with a broad selection of collar heights to match the soft tissue thickness. Available virtually for all implant platforms and are intended to be connectors between dental implants and multiple implant screw retained restorations. Multiunit abutments are indicated in all screw retained full arch prosthesis, correcting implant angulations and height disparities to level restorative platform for the prosthesis. Multiunit abutments extend benefits of easy and predictable seating of restoration, provide passive draw, reduce stress translated into restorative system due to the passive nature of the seating process, easy retrival and replacing of prosthesis during patient follow ups. The only limitaion is that the screw 
that holds bridge on is quite tiny, that one cannot tighten the screw down very much because it is so small.13,14

Selection of implant abutment is also governed by several other factors, implant position, implant angulation, hard and soft tissue profile, smile line, crown height space, hygiene access and occlusion.

\section{Implant Position}

Impant position refers to position of implant in relation to adjacent teeth and the final prosthesis. Less than an ideal implant postion can be corrected by cement retained, angulated and custom abutments. Severely affected implant position as in bone dehiscence, implant positioned too far buccaly or lingually cannot be corrected with these abutments and restoration becomes difficult. 15,16

Crown height space is measured from the crest of the ridge to plane of occlusion in posterior region and incisal edge in anterior region. Insufficient crown height space can be managed by screw retained, custsomised and metal abutments and in cases with excessive crown height space, prefabricated and customised abutments can be used. ${ }^{15}$

\section{Smile Line / Hard and Soft Tissue Profile}

In patients with high smile line ceramic or zirconia abutments can be used. Customised abutments can be contoured to follow ginigival architecture. Abutment collar design is chosen based on height, depth and contour of soft tissue to achieve desired emergence profile. Pink porcelin recreates missing pink component in hard and soft tissue defects to enhance esthetics. ${ }^{15,17}$

\section{CONCLUSIONS}

In situations with high esthetic demands (high smile line), with anatomically demanding factors (high scalloping of soft tissues) and low mechanical challenges (less occlusal forces, regular implant platform) customised all ceramic implant abutments are recommended. In situations with low esthetic demands and mechanically demanding conditions (high occlusal forces, narrow diameter platform) metallic abutments are recommended. In high esthetic demanding cases that are also mechanically challenging, patient should be informed about the decision to be made between, more esthetic solution with compromised strength (all ceramic) or possible esthetic challenge delivering mechanical stability (metallic abutments). In such cases soft tissue thickness may be increased to reduce the risk of soft tissue discoloration. ${ }^{7}$

Titanium based hybrid abutments can deliver combination of advantages of all ceramic and metallic abutments, with improved esthetics as well as mechanical stability. In cement retained restorations, use of custom abutments provide better solution with an individualized cement margin linet and better soft tissue response. ${ }^{7}$

Various options are available to prosthetically restore implants; there is no single ideal solution. Selection of type of the abutment for a specific case lies solely on the practitioner based on his assessment of case in terms of the severity of the angulations, esthetic requirements, axial load distribution, soft tissue profile etc. The most favourable option can be chosen for the prosthodontic management of the osseointegrated implants. Abutment selection should be considered as a part of initial treatment planning procedure.

Financial or other competing interests: None.

Disclosure forms provided by the authors are available with the full text of this article at jemds.com.

\section{REFERENCES}

[1] Misch CE. Dental implant prosthetics. $2^{\text {nd }}$ edn. St. Louis: Elsevier Mosby 2005.

[2] Karunagaran S, Paprocki GJ, Wicks R, Markose S. A review of implant abutments--abutment classification to aid prosthetic selection. J Tenn Dent Assoc 2013;93(2):18-24.

[3] Shah RM, Aras MA, Chitre V. Implant abutment selection: a literature review. Int $J$ Oral Implantol Clin Res 2014;5(2):43-9.

[4] Piermatti J. Considerations in abutment selection. Dent Today 2017;36(3):74-5.

[5] Narang P, Gupta H, Arora A, et al. Biomechanics of implant abutment connection: a review. Indian J Stomatol 2011;2(2):113-6.

[6] Shadid R, Sadaqa N. A comparison between screw- and cement-retained implant prostheses. A literature review. J Oral Implantol 2012;38(3):298-307.

[7] Zarauz C, Pitta J, Pradies G, et al. Clinical recommendations for implant abutment selection for single-implant reconstructions: customized vs. standardized ceramic and metallic solutions. Int J Periodontics Restorative Dent 2020;40(1):31-7.

[8] Wittneben JG, Joda T, Weber HP, et al. Screw retained vs. cement retained implant-supported fixed dental prosthesis. Periodontol 2000. 2017;73(1):141-51.

[9] Bharate V, Kumar Y, Koli D, et al. Effect of different abutment materials (zirconia or titanium) on the crestal bone height in 1 year. J Oral Biol Craniofac Res 2020;10(1):372-4.

[10] https: / / pocketdentistry.com

[11] Osorio J, Kerstein RB. Use of CAD-CAM technology in custom abutment manufacturing. In: Shafie HR, edr. Clinical and laboratory manual of dental implant abutments. John Wiley \& Sons, Inc., 2014: p. 65-101.

[12] Rani S, Devi J, Jain C, et al. Esthetic rehabilitaion of anterior eeth with copy milled restorations: a report of two cases. Case Rep Dent 2017;2017:2841398.

[13] Kosinski TF. chairside@glidewelldental.com/May 10, 2019.

[14] Buzayan MM, Yunus NB. Passive fit in screw retained multi-unit implant prosthesis understanding and achieving: a review of the literature. J Indian Prosthodont Soc 2014;14(1):16-23.

[15] Bhavana BL, Rahul N, Fouzia B, et al. Abutment options for the restoration of the maligned implants: a review. IJSS Case Reports \& Reviews 2016;2(12):22-6.

[16] Rathee M, Bhoria M, Boora P. An insight into dental implant abutment selection criteria: an overview. J Adv Oral Res 2014;5(3):1-4.

[17] Sonune SJ, Kumar S, Jadhav MS, et al. Gingival-colored porcelain: a clinical report of an esthetic-prosthetic paradigm. Int J Appl Basic Med Res 2017;7(4):275-7. 